\title{
Almost all positive continuous linear functionals can be extended
}

\author{
Josse van Dobben de Bruyn ${ }^{1}$
}

Received: 2 May 2021 / Accepted: 18 November 2021 / Published online: 19 February 2022

(c) The Author(s) 2022

\begin{abstract}
Let $F$ be an ordered topological vector space (over $\mathbb{R}$ ) whose positive cone $F_{+}$is weakly closed, and let $E \subseteq F$ be a subspace. We prove that the set of positive continuous linear functionals on $E$ that can be extended (positively and continuously) to $F$ is weak-* dense in the topological dual wedge $E_{+}^{\prime}$. Furthermore, we show that this result cannot be generalized to arbitrary positive operators, even in finite-dimensional spaces.
\end{abstract}

Keywords Convex cone $\cdot$ Partially ordered topological vector space $\cdot$ Continuous positive linear functional $\cdot$ Positive extension

Mathematics Subject Classification 46A40 · 47B65 - 47L07 · 52A20

Extension theorems for positive operators have been studied in great detail, and can be found in many textbooks on ordered vector spaces (e.g. [11,§V.5], [1,§1.4-1.5]). Many classical extension theorems provide sufficient conditions for a given operator to have a positive extension. These include a classical extension theorem of Kantorovich (see [1, Theorem 1.30]), and a theorem of Lotz on positive operators from a Banach sublattice of a Banach lattice to an AL-space (see [6,Proposition 3.2], [10,Theorem II.8.9]).

In general, not every positive operator can be extended, and the problem already arises when looking at functionals. In 1957, Mirkil proved that, for a finite-dimensional ordered vector space $F$ whose positive cone $F_{+}$is closed, all positive linear functionals on all subspaces of $F$ can be extended if and only if $F_{+}$is polyhedral (see [7,Corollary 1], [4,Theorem 4.13]). That same year, Bauer and Namioka independently found a necessary and sufficient condition for a positive continuous linear functional $E \rightarrow$

Partially supported by the Dutch Research Council (NWO), project number 613.009.127.

$凶$ Josse van Dobben de Bruyn

J.vanDobbendeBruyn@tudelft.nl

1 Delft Institute of Applied Mathematics, Delft University of Technology, Mekelweg 4, 2628 CD Delft, The Netherlands 
$\mathbb{R}$ defined on a subspace $E$ of a topological vector space $F$ to have a positive and continuous extension to $F$ (see [3,Theorem 1], [8, Theorem 4.4], [11, Theorem V.5.4]).

In this note, we look at things from a different perspective. Instead of determining whether or not a positive operator can be extended, we try to approximate it by extendable positive operators. We prove that the extendable positive linear functionals are weak-* dense in the dual wedge. Furthermore, we show that this result cannot be extended to arbitrary positive operators, even if the spaces are finite-dimensional.

Although the proofs in this note are relatively simple, the results appear to be unknown in the ordered vector spaces community. In particular, the author has not been able to locate an earlier proof (or statement) of Theorem 1 in the literature.

We follow notation and terminology from [1].

For positive functionals, we have the following positive result.

Theorem 1 Let $F$ be a preordered topological vector space, and let $E \subseteq F$ be a subspace. If the topological dual $F^{\prime}$ separates points on $F$, and if the positive wedge $F_{+}$is weakly closed, then the set of all positive continuous linear functionals on $E$ that can be extended positively and continuously to $F$ is weak-* dense in $E_{+}^{\prime}$.

We make two remarks about the statement of Theorem 1. First, if $F$ is locally convex, then we may replace the requirement that $F_{+}$is weakly closed by the requirement that $F_{+}$is closed. These two requirements are now equivalent, because $F_{+}$is convex.

Second, if $F_{+}$is weakly closed and if $F_{+} \cap-F_{+}=\{0\}$, then $\{0\}$ is also weakly closed, so the weak topology is Hausdorff. Hence, if $F_{+}$is a cone, then the requirement that $F^{\prime}$ separates points on $F$ is automatically met. Thus, the statement from the abstract is recovered.

Proof of Theorem 1 Define $\mathcal{R}:=\left\{\left.\varphi\right|_{E}: \varphi \in F_{+}^{\prime}\right\} \subseteq E_{+}^{\prime}$, and note that $\mathcal{R}$ is the wedge of positive continuous linear functionals $E \rightarrow \mathbb{R}$ that can be extended positively and continuously to $F$. Since $F_{+}$is weakly closed, we have

$$
F_{+}=\left\{x \in F:\langle x, \varphi\rangle \geq 0 \text { for all } \varphi \in F_{+}^{\prime}\right\}
$$

It follows that

$$
E_{+}:=F_{+} \cap E=\left\{x \in E:\langle x, \varphi\rangle \geq 0 \text { for all } \varphi \in F_{+}^{\prime}\right\} .
$$

This shows that $E_{+}$is the predual wedge of $\mathcal{R}$. Hence, by the bipolar theorem, $E_{+}^{\prime}$ is the weak-* closure of $\mathcal{R}$.

A little more can be said if the subspace $E \subseteq F$ is finite-dimensional, as the following corollaries show.

Corollary 2 Let $F$ be a preordered topological vector space whose positive wedge $F_{+}$ is weakly closed, let $E \subseteq F$ be a finite-dimensional subspace, and let $\lambda$ denote the Lebesgue (i.e. Haar) measure on $E^{*}$. Then $\lambda$-almost all positive linear functionals $E \rightarrow \mathbb{R}$ can be extended to a positive continuous linear functional $F \rightarrow \mathbb{R}$. 
Proof The set $\mathcal{R} \subseteq E_{+}^{*}$ of extendable positive linear functionals is a convex set, and it follows from Theorem 1 that $\overline{\mathcal{R}}=E_{+}^{*}$. The result follows since $E_{+}^{*} \backslash \mathcal{R}=\overline{\mathcal{R}} \backslash \mathcal{R} \subseteq \partial \mathcal{R}$, and the boundary of a convex set in Euclidean space has Lebesgue measure zero (see e.g. [5]).

Corollary 3 Let $F$ be a preordered topological vector space whose positive wedge $F_{+}$is weakly closed, and let $E \subseteq F$ be a finite-dimensional subspace. Then every strictly positive linear functional on $E$ can be extended to a positive continuous linear functional on $F$.

If, in addition, $F$ is finite-dimensional and $F_{+} \cap-F_{+}=\{0\}$, then the extension can be chosen to be strictly positive as well.

Proof If $E_{+} \cap-E_{+} \neq\{0\}$, then $E$ does not have strictly positive linear functionals, so the statement is vacuously true. Assume henceforth that $E_{+} \cap-E_{+}=\{0\}$. Then $E_{+}$ is a closed cone, so the dual wedge $E_{+}^{*}$ is generating. Therefore $E_{+}^{*}$ has non-empty interior, and the strictly positive linear functionals on $E$ are precisely the interior points of $E_{+}^{*}$ (see [1,Lemma 2.17]).

By Theorem 1, the convex set $\mathcal{R}$ of extendable positive linear functionals is dense in $E_{+}^{*}$. It follows from finite-dimensional convex geometry that $\mathcal{R}$ and $E_{+}^{*}$ have the same interior points (see [9,Theorem 6.3]). Hence, for every strictly positive linear functional $\varphi: E \rightarrow \mathbb{R}$, we have $\varphi \in \operatorname{int}\left(E_{+}^{*}\right)=\operatorname{int}(\mathcal{R}) \subseteq \mathcal{R}$, which shows that $\varphi$ is extendable.

Assume now that $F$ is finite-dimensional and that $F_{+}$is a cone. Then $F_{+}^{*}$ also has non-empty interior, and its interior points are precisely the strictly positive linear functionals on $F$. Now we use the fact that a linear map $T$ between finite-dimensional spaces preserves the relative interior of every convex set $M$; that is, $\operatorname{relint}(T[M])=$ $T$ [relint $(M)]$ (see [9,Theorem 6.6]). Since $\mathcal{R}$ is the image of $F_{+}^{*}$ under the canonical map $F^{*} \rightarrow F^{*} / E^{\perp} \cong E^{*}$, and since $\mathcal{R}$ and $F_{+}^{*}$ are full-dimensional, it follows that every interior point of $\mathcal{R}$ is the image of an interior point of $F_{+}^{*}$. Equivalently, every strictly positive linear functional $E \rightarrow \mathbb{R}$ can be extended to a strictly positive linear functional $F \rightarrow \mathbb{R}$.

One might ask if Theorem 1 remains true if the positive linear functionals are replaced by positive operators. Unfortunately, this is not the case, even if the spaces are finite-dimensional, as we will now demonstrate.

A wedge $\mathcal{K} \subseteq E$ is a simplex cone (or Yudin cone) if there is an algebraic basis $\mathcal{B}$ of $E$ such that $\mathcal{K}$ is the wedge generated by $\mathcal{B}$.

If $E$ and $G$ are vector spaces, and if $\varphi \in E^{*}$ and $z \in G$, then we write $z \otimes \varphi$ for the linear map $E \rightarrow G, x \mapsto\langle x, \varphi\rangle z$.

For our counterexample, we will consider the following situation.

Situation 4 Let $E$ be a finite-dimensional vector space, and let $E_{+} \subseteq E$ be a generating polyhedral cone which is not a simplex cone. Let $\varphi_{1}, \ldots, \varphi_{m} \in E_{+}^{*}$ be representatives of the extremal rays of $E_{+}^{*}$. Then $E_{+}=\bigcap_{i=1}^{m}\left\{x \in E:\left\langle x, \varphi_{i}\right\rangle \geq 0\right\}$, and every positive linear functional is a positive combination of $\varphi_{1}, \ldots, \varphi_{m}$. Additionally, let $F:=\mathbb{R}^{m}$ with the standard cone $F_{+}:=\mathbb{R}_{\geq 0}^{m}$, so that the map $T: E \rightarrow F, x \mapsto$ $\left(\varphi_{1}(x), \ldots, \varphi_{m}(x)\right)$ is bipositive. We will identify $E$ with a subspace of $F$ via this map. 
Proposition 5 In Situation 4, the positive linear maps $E \rightarrow E$ that can be extended to a positive linear map $F \rightarrow E$ are precisely the maps of the form $\sum_{i=1}^{k} x_{i} \otimes \psi_{i}$ with $x_{1}, \ldots, x_{k} \in E_{+}$and $\psi_{1}, \ldots, \psi_{k} \in E_{+}^{*}$.

Proof Let $e_{1}, \ldots, e_{m}$ denote the standard basis of $F=\mathbb{R}^{m}$. If $T: E \rightarrow E$ is a positive linear map that can be extended to a positive linear map $S: F \rightarrow E$, then we have

$$
T(x)=S\left(\varphi_{1}(x), \ldots, \varphi_{m}(x)\right)=S\left(e_{1}\right) \varphi_{1}(x)+\cdots+S\left(e_{m}\right) \varphi_{m}(x),
$$

so $T$ can be written as $T=\sum_{j=1}^{m} S\left(e_{j}\right) \otimes \varphi_{j}$.

Conversely, suppose that $T=\sum_{i=1}^{k} x_{i} \otimes \psi_{i}$ with $x_{1}, \ldots, x_{k} \in E_{+}$and $\psi_{1}, \ldots, \psi_{k} \in E_{+}^{*}$. Every $\psi_{i}$ can be written as a positive combination of the $\varphi_{1}, \ldots, \varphi_{m}$, so after rearranging the terms we may write $T=\sum_{j=1}^{m} y_{j} \otimes \varphi_{j}$, where the $y_{j}$ are positive combinations of the $x_{i}$. In particular, $y_{1}, \ldots, y_{m} \in E_{+}$. Therefore the map $S: F \rightarrow E, e_{j} \mapsto y_{j}$ is a positive extension of $T$.

The following theorem of Barker and Loewy tells us that approximation by operators of the form described in Proposition 5 is not always possible.

Theorem 6 [Barker-Loewy, [2,Proposition 3.1]] Let $E$ be a finite-dimensional ordered vector space whose positive cone $E_{+}$is closed and generating. Then the identity $\operatorname{id}_{E}: E \rightarrow E$ can be written as the limit of a sequence of operators of the form $\sum_{i=1}^{k} x_{i} \otimes \psi_{i}$ with $x_{1}, \ldots, x_{k} \in E_{+}$and $\psi_{1}, \ldots, \psi_{k} \in E_{+}^{*}$ if and only if $E_{+}$is a simplex cone.

If $E$ and $F$ are as in Situation 4, then it follows from Proposition 5 and Theorem 6 that the identity $\operatorname{id}_{E}: E \rightarrow E$ cannot be approximated by positive operators that can be extended to positive operators $F \rightarrow E$.

Remark 7 Although Theorem 6 is exactly what Barker and Loewy proved and exactly what we used, we should point out that Tam later showed that the cone of operators of the form $\sum_{i=1}^{k} x_{i} \otimes \psi_{i}$ with $x_{1}, \ldots, x_{k} \in E_{+}$and $\psi_{1}, \ldots, \psi_{k} \in E_{+}^{*}$ is already closed (see [12,Theorem 1]), and used this to give a simpler proof of Theorem 6 (see [12, Theorem 4]). As a result, another equivalent condition in Theorem 6 is that $\mathrm{id}_{E}$ itself can be written in this positive tensor form, rather than being a limit of operators of this form.

In Situation 4, an easier way to see that the cone generated by $\{x \otimes \psi: x \in$ $\left.E_{+}, \psi \in E_{+}^{*}\right\}$ is closed is to note that it is finitely generated. In any case, we conclude that the positive operators $E \rightarrow E$ which can be extended to a positive operator $F \rightarrow E$ form a closed cone which is strictly contained in the (closed) cone of all positive operators $E \rightarrow E$.

Acknowledgements The author wishes to thank Marcel de Jeu for helpful comments and suggestions, which helped improve the clarity and brevity of this note.

Open Access This article is licensed under a Creative Commons Attribution 4.0 International License, which permits use, sharing, adaptation, distribution and reproduction in any medium or format, as long as you give appropriate credit to the original author(s) and the source, provide a link to the Creative Commons licence, 
and indicate if changes were made. The images or other third party material in this article are included in the article's Creative Commons licence, unless indicated otherwise in a credit line to the material. If material is not included in the article's Creative Commons licence and your intended use is not permitted by statutory regulation or exceeds the permitted use, you will need to obtain permission directly from the copyright holder. To view a copy of this licence, visit http://creativecommons.org/licenses/by/4.0/.

\section{References}

1. Aliprantis, C.D., Tourky, R.: Cones and Duality. Graduate Studies in Mathematics, vol. 84. American Mathematical Society, Providence (2007)

2. Barker, G.P., Loewy, R.: The structure of cones of matrices. Linear Algebra Appl. 12(1), 87-94 (1975)

3. Bauer, H.: Sur le prolongement des formes lineáires positives dans un espace vectoriel ordonné. Comptes rendus hebdomadaires des séances de l'Académie des Sciences 244(1), 289-292 (1957)

4. Klee, V.: Some characterizations of convex polyhedra. Acta Math. 102(1-2), 79-107 (1959)

5. Lang, R.: A note on the measurability of convex sets. Archiv der Math. 47(1), 90-92 (1986)

6. Lotz, H.P.: Extensions and liftings of positive linear mappings on Banach lattices. Trans. Am. Math. Soc. 211, 85-100 (1975)

7. Mirkil, H.: New characterizations of polyhedral cones. Can. J. Math. 9, 1-4 (1957)

8. Namioka, I.: Partially ordered linear topological spaces, Memoirs of the American Mathematical Society, no. 24 (1957)

9. Rockafellar, R.T.: Convex Analysis. Princeton University Press, Princeton (1970)

10. Schaefer, H.H.: Banach Lattices and Positive Operators. Grundlehren der mathematischen Wissenschaften, vol. 215. Springer, Berlin (1974)

11. Schaefer, H.H., Wolff, M.P.: Topological Vector Spaces. Graduate Texts in Mathematics, vol. 3, 2nd edn. Springer, Berlin (1999)

12. Tam, B.-S.: Some results of polyhedral cones and simplicial cones. Linear Multilinear Algebra 4(4), 281-284 (1977)

Publisher's Note Springer Nature remains neutral with regard to jurisdictional claims in published maps and institutional affiliations. 